DOI: https://doi.org/10.24297/jam.v16i0.8016

\title{
CONGRUENCES ON *-SIMPLE TYPE A I-SEMIGROUPS
}

\author{
${ }^{1}$ Ndubuisi R.U, ${ }^{2}$ Asibong-Ibe U.I, ${ }^{3}$ Udoaka O.G \\ 1, 2Department of Mathematics \& Statistics, University of Port Harcourt, Port Harcourt, Nigeria. \\ ${ }^{3}$ Department of Mathematics \& Statistics, Akwa Ibom State University, Ikot Akpaden, Nigeria. \\ u_ndubuisi@yahoo.com
}

\begin{abstract}
This paper obtains a characterisation of the congruences on *-simple type A I-semigroups. The *-locally idempotent-separating congruences, strictly *-locally idempotent-separating congruences and minimum cancellative monoid congruences, are characterised.
\end{abstract}

Keywords: Type A I-Semigroup, *-Locally Idempotent-Separating, Cancellative Monoid Congruence, Generalized Bruck-Reilly *-Extensions.

\section{Introduction}

For a semigroup $S, E(S)$ will denote the set of idempotents of $S$. If $S$ is a semigroup with non-empty set of idempotents $E(S)$, we define a partial order " $\leq$ " on $\mathrm{E}(\mathrm{S})$ such that $e \leq f$ if and only if $e f=f e=e$. Let $I$ denote the set of all integers and let $\mathbb{N}^{0}$ denote the set of non-negative integers. A semigroup $S$ is said to be an $I^{-}$ semigroup if and only if $E(S)$ is order isomorphic to $I$ under the reverse of the partial order.

The structure theorem for *-simple type A I-semigroups was established in [8], as an extension of the structure theorem for simple I-inverse semigroups and *-simple type A $\omega$-semigroups due to Warne [10] and AsibongIbe [1]. This paper is a follow up of the study of congruences on *-bisimple type A I-semigroups studied by Ndubuisi and Asibong-lbe [7], where the congruences were identified as idempotent-separating congruence and minimum cancellative monoid congruence.

Earlier investigations in [6] and [10] studied congruences on *-simple type A $\omega$-semigroups and congruences on simple I-inverse semigroups respectively. Determination of congruences throughout this paper is based on their description in [6].

This work is divided as follows. Section 2 contains a minimum of results concerning *-simple type A Isemigroups. The content of section 3 is a determination of *-locally idempotent-separating congruences, strictly *-locally idempotent-separating congruences and minimum cancellative monoid congruences of a *-simple type A I-semigroup.

Let us recall some definitions which will be useful in the study.

Let $S$ be a semigroup and let $a, b \in S$. Then the elements $a$ and $b$ are said to to be $\mathcal{R}^{*}$-related written $a \mathcal{R}^{*} b$ if and only if for all $x, y \in S^{1}, x a=y a$ if and only if $x b=y b$. The relation $\mathcal{L}^{*}$ is defined

dually. The join of the equivalence relations $\mathcal{R}^{*}$ and $\mathcal{L}^{*}$ is denoted by $\mathcal{D}^{*}$ and their intersection by $\mathcal{H}^{*}$. Thus $a \mathcal{H}^{*} b$ if and only if $a \mathcal{R}^{*} b$ and $a \mathcal{L}^{*} b$. In general $\mathcal{R}^{*} \circ \mathcal{L}^{*} \neq \mathcal{L}^{*} \circ \mathcal{R}^{*}$ as shown in [3].

Following Fountain [4] a semigroup is an abundant semigroup if every $\mathcal{L}^{*}$-class and every $\mathcal{R}^{*}$-class in $S$ contain idempotents. An abundant semigroup $S$ is adequate [3] if $E(S)$ forms a semilattice. In an adequate semigroup every $\mathcal{L}^{*}$-class $\mathcal{R}^{*}$-class contains a unique idempotent. 
Let $a$ be an element of an adequate semigroup $S$, and $a^{*}\left(a^{\dagger}\right)$ denotes the unique idempotent in the $\mathcal{L}^{*}$-class $L_{a}^{*}\left(\mathcal{R}^{*}\right.$-class $\left.R_{a}^{*}\right)$ containing $a$.

We remark that a type A (in particular, right type A) semigroup realized in Fountain [2] as a special type of right PP monoid with e-cancellable element where $e \in E(S)$, the set of idempotents in $S$. An adequate semigroup $S$ is said to be a type A semigroup if $e a=a(e a)^{*}$ and $a e=(a e)^{\dagger} a$ for all $a \in S$ and $e \in E(S)$.

We conclude this section by defining the relation $\mathcal{J}^{*}$. Let $S$ be a semigroup and $I^{*}$ be an ideal of $S$. Then $I^{*}$ is said to be a *-ideal if $L_{a}^{*} \subseteq I^{*}$ and $R_{a}^{*} \subseteq I^{*}$ for all $a \in I^{*}$. The smallest *-ideal containing ' $a$ ' is the principal *-ideal generated by ' $a$ ' and is denoted by $J^{*}(a)$. For $a, b \in S, a \mathcal{J}^{*} b$ if and only if $J^{*}(a)=J^{*}(b)$. The relations $\mathcal{J}^{*}$ contains $\mathcal{D}^{*}$.

A semigroup $S$ is said to be *-simple if the only *-ideal of $S$ is itself. Clearly a semigroup is *-simple if all its elements are $\mathcal{J}^{*}$-related. To have a clear picture of $\mathcal{J}^{*}$-related elements we recall the following Lemma.

Lemma 1.1 [3]. Let $S$ be a semigroup and $a, b \in S$. Then $b \in J^{*}(a)$ if and only if there are elements $a_{0}, a_{1}, \ldots, a_{n} \in S, x_{1}, x_{2}, \ldots, x_{n}, y_{1}, y_{2}, \ldots, y_{n} \in S^{1}$ such that $a=a_{0}, b=a_{n}$ and $a_{i} \mathcal{D}^{*} x_{i} a_{i-1} y_{i}$, for $i=1,2, \ldots, n$.

Other basic results discussed in [3] will be assumed. The notation adopted in this paper is similar to that in Fountain [3], Howie [5], Asibong-Ibe [1] and Makanjuola [6].

Recently type A semigroups have been shown to be special type of restriction semigroups. In this case type A $\omega$-semigroup will essentially be an $\omega$-restriction semigroups. The idea developed here will prove useful in the study of restriction semigroups.

However, we will in this work retain the term type A semigroups generally.

\section{2. *-Simple Type A I-Semigroups}

Following [9], let $T=\bigcup_{i=0}^{d-1} M_{i}$ be a chain of cancellative monoids. Each element $x_{i} \in T$ is necessarily in $M_{i}$ for $0 \leq i \leq d-1$. An identity $e_{i} \in M_{i}$ is an idempotent in $T$. Clearly $e_{i} \in T$ form a chain of idempotents $e_{0}>e_{i}>$ $\cdots>e_{d-1}$.

Let $\theta: T \rightarrow M_{0}$ be a monoid morphism and let $S=T \times I \times I$ (where $I$ is the set of all integers) be the set of all ordered triples $\left(x_{i}, m, n\right)$ where $m \in \mathbb{N}^{0}, n \in I, 0 \leq i \leq d-1$ and $x_{i} \in T$.

Define multiplication on $\mathrm{S}$ by the rule

$$
\left(x_{i}, m, n\right)\left(y_{j}, p, q\right)= \begin{cases}\left(x_{i} \cdot f_{n-p, p}^{-1} \cdot y_{j} \theta^{n-p} \cdot f_{n-p, q}, m, n+q-p\right) & \text { if } n \geq p \\ \left(f_{p-n, m}^{-1} \cdot x_{i} \theta^{p-n} \cdot f_{p-n, n} \cdot y_{j}, m+p-n, q\right) & \text { if } n \leq p\end{cases}
$$

where $\theta^{0}$ is the identity automorphism of $T$, and for $m \in \mathbb{N}^{0}, n \in I, f_{0, n}=e_{i}$, the identity of $M_{i}$, while for $m>$ $0, f_{m, n}=u_{n+1} \theta^{m-1} \cdot u_{n+2} \theta^{m-2} \ldots u_{n+(m-1)} \theta \cdot u_{n+m}$, and

$f_{m, n}^{-1}=u_{n+m}^{-1} \cdot u_{n+(m-1)}^{-1} \theta \ldots u_{n+2}^{-1} \theta^{m-2} \cdot u_{n+1}^{-1} \theta^{m-1}$, where $\left\{u_{n}: n \in I\right\}$ is a collection of $\mathrm{T}$ with $u_{n}=e_{i}$ for $n>0$. Denote a semigroup formed by $S=G B R^{*}(T, \theta)$ where $T=\cup_{i=0}^{d-1} M_{i}$.

If for each $i$ we now let $M_{i}=\left\{e_{i}\right\}$, a monoid with one element, we obtain the set $I \times I$ under the multiplication

$$
(m d+i, n d+i)(p d+j, q d+j)= \begin{cases}(m d+i,(n+q-p) d+i) & \text { if } n \geq p \\ ((m+p-n) d+j, q d+j) & \text { if } n \leq p\end{cases}
$$


We denote $I \times I$ under the above multiplication by $B_{d}^{*}$ and call it the extended bicyclic semigroup.

If we let $\left(x_{i}, m, n\right)$ be an idempotent in $S$. Then

$\left(x_{i}, m, n\right)=\left(x_{i}, m, n\right)\left(x_{i}, m, n\right)$
$\left\{\begin{array}{cc}\left(x_{i} \cdot f_{n-m, m}^{-1} \cdot x_{i} \theta^{n-m} \cdot f_{n-m, n}, m, n-m+n\right) & \text { if } n \geq m \\ \left(f_{m-n, m}^{-1} \cdot x_{i} \theta^{m-n} \cdot f_{m-n, n} \cdot x_{i}, m-n+m, n\right) & \text { if } n \leq m\end{array}\right.$

in which case $m=n, x_{i}^{2}=x_{i}$.

Conversely, suppose $x_{i}^{2}=x_{i}$ then we have that $\left(x_{i}, m, n\right)\left(x_{i}, m, n\right)=\left(x_{i}, m, n\right)$. Thus $\left(x_{i}, m, n\right)$ is an idempotent if and only if $m=n$ and $x_{i}$ is an idempotent in $S$.

The following results were proved in [8].

Lemma 2.1. Let $S=G B R^{*}(T, \theta)$ be a generalized Bruck-Reilly *-extension of a monoid $T$, where $T=\cup_{i=0}^{d-1} M_{i}$ is a finite chain of cancellative monoids $M_{i}$. Let $\left(x_{i}, m, n\right),\left(y_{j}, p, q\right) \in S$. Then

i) $\left(x_{i}, m, n\right) \mathcal{R}^{*}\left(y_{j}, p, q\right)$ if and only if $m=p$ and $i=j$.

ii) $\left(x_{i}, m, n\right) \mathcal{L}^{*}\left(y_{j}, p, q\right)$ if and only if $n=q$ and $i=j$.

iii) $\left(x_{i}, m, n\right) \mathcal{J}^{*}\left(y_{j}, p, q\right)$. That is $S$ is *-simple.

Lemma 2.2. $S=G B R^{*}(T, \theta)$ is a type A semigroup if and only $T$ is a type A semigroup

Theorem 2.3. Let $S=G B R^{*}(T, \theta)$ be the generalized Bruck-Reilly *-extension of the monoid $T$ where $T=$ $\cup_{i=0}^{d-1} M_{i}$. Then $S$ is a *-simple type A I-semigroup with $d \mathcal{D}^{*}$-classes.

We conclude this section, with the structure theorem of *-simple type A I-semigroups.

Theorem 2.4 [8]. Let $S$ be a *-simple type A I-semigroup with $d \mathcal{D}^{*}$-classses. Then $S$ is isomorphic to a generalized Bruck-Reilly *-extension $S=G B R^{*}(T, \theta)$ of a monoid $T$, where $T=\bigcup_{i=0}^{d-1} M_{i}$ is a finite chain of cancellative monoids $M_{i}$ and $\theta$ is an endomorphism of $T$ with image in $M_{0}$.

\section{The Congruences}

In this section, we will determine the congruence relations on a *-simple type A $I$-semigroup $S=G B R^{*}(T, \theta)$. We first present the properties of the congruences and then show that every congruence relation $\rho$ on $S$ is either a *-locally idempotent-separating congruence (if no two distinct $\mathcal{D}^{*}$-related idempotents are $\rho$-related) or all the idempotents are in one $\rho$-class. We also provide a method for constructing the strictly *-locally idempotent separating congruences. Lastly, we show that there is a minimum cancellative monoid congruence on $S$.

Lemma 3.1. Let $S=G B R^{*}(T, \theta)$ be a ${ }^{*}$-simple type A I-semigroup where $T=\bigcup_{i=0}^{d-1} M_{i}$ is a semilattice of cancellative monoids. Then $\mathcal{H}^{*}$ is a congruence on $S$, and $S / \mathcal{H}^{*} \cong B_{d}^{*}$.

Proof. The mapping $\theta: S \rightarrow B_{d}^{*}$ by

$$
\left(x_{i}, m, n\right) \theta=(m d+i, n d+i)
$$

is onto. It is a homomorphism since 


$$
\begin{aligned}
\left(\left(x_{i}, m, n\right)\left(y_{j}, p, q\right)\right) \theta=\left\{\begin{array}{ll}
\left(x_{i} \cdot f_{n-p, p}^{-1} \cdot y_{j} \theta^{n-p} \cdot f_{n-p, q}, m, n+q-p\right) & \text { if } n \geq p \\
\left(f_{p-n, m}^{-1} \cdot x_{i} \theta^{p-n} \cdot f_{p-n, n} \cdot y_{j}, m+p-n, q\right) & \text { if } n \leq p
\end{array} \times \theta\right. & \text { if } n \geq p \\
= & \begin{cases}\left(x_{i} \cdot f_{n-p, p}^{-1} \cdot y_{j} \theta^{n-p} \cdot f_{n-p, q}, m, n+q-p\right) \theta & \text { if } n \leq p \\
\left(f_{p-n, m}^{-1} \cdot x_{i} \theta^{p-n} \cdot f_{p-n, n} \cdot y_{j}, m+p-n, q\right) \theta & \text { if } n \geq p\end{cases} \\
= & \text { if } n \leq p \\
((m d+i,(n+q-p) d+i) & \\
= & (m d+i, n d+i)(p d+j, q d+j) \\
= & \left(x_{i}, m, n\right) \theta\left(y_{j}, p, q\right) \theta .
\end{aligned}
$$

Thus $\theta$ is a homomorphism.

Furthermore, $\left(\left(x_{i}, m, n\right)\left(y_{j}, p, q\right)\right) \in \mathcal{H}^{*}$ if and only if $(m d+i, n d+i)=(p d+j, q d+j)$; hence $\theta \circ \theta^{-1}=\mathcal{H}^{*}$ and the result follows.

Lemma 3.2. Let $\rho$ be a congruence on a *-simple type A $I$-semigroup $S=G B R^{*}(T, \theta)$ where $T=\bigcup_{i=0}^{d-1} M_{i}$. Suppose that

(i) $\left(e_{i}, m, m\right) \rho\left(e_{j}, m, m\right)$ then for any $n \in I,\left(e_{i}, n, n\right) \rho\left(e_{j}, n, n\right)$

(ii) $\left(e_{i}, m, m\right) \rho\left(e_{j}, m+1, m+1\right)$ then for any $n \in I,\left(e_{i}, n, n\right) \rho\left(e_{j}, n+1, n+1\right)$

Proof. i) Let $\left(e_{0}, n, m\right),\left(e_{0}, m, n\right) \in S$, then

$$
\begin{gathered}
\left(e_{0}, n, m\right)\left(e_{i}, m, m\right)\left(e_{0}, m, n\right)=\left(e_{i}, n, n\right) \\
\left(e_{0}, n, m\right)\left(e_{j}, m, m\right)\left(e_{0}, m, n\right)=\left(e_{j}, n, n\right)
\end{gathered}
$$

and

$$
\begin{gathered}
\left(e_{0}, m, n\right)\left(e_{i}, n, n\right)\left(e_{0}, n, m\right)=\left(e_{i}, m, m\right) \\
\left(e_{0}, m, n\right)\left(e_{j}, n, n\right)\left(e_{0}, n, m\right)=\left(e_{j}, m, m\right) .
\end{gathered}
$$

ii) Let $\left(e_{0}, n, m\right),\left(e_{0}, m, n\right) \in S$, then we have

$$
\begin{aligned}
& \left(e_{0}, n, m\right)\left(e_{i}, m, n\right)=\left(e_{i}, n, n\right) . \\
& \left(e_{0}, n, m\right)\left(e_{j}, m+1, m+1\right)\left(e_{0}, m, n\right)=\left(e_{j}, n+1, n+1\right),
\end{aligned}
$$

and

$$
\begin{aligned}
& \left(e_{0}, m, n\right)\left(e_{i}, n, n\right)\left(e_{0}, n, m\right)=\left(e_{i}, m, m\right) . \\
& \left(e_{0}, m, n\right)\left(e_{j}, n+1, n+1\right)\left(e_{0}, n, m\right)=\left(e_{j}, m+1, m+1\right) .
\end{aligned}
$$

Hence the proof.

We now establish an important property of congruences on *-simple type A $I$-semigroups. 
Theorem 3.3. A congruence $\rho$ on a *-simple type A l-semigroup is either a *-locally idempotent-separating congruence or all the idempotents are in one $\rho$-class.

Proof. Suppose that the idempotent elements of $S$ are not in one $\rho$-class and $e_{m d+i} \rho e_{(m+k) d+i}$ for some $m, k \in I, k>0$ and $0 \leq i \leq d-1$. We are to show that no two distinct $\mathcal{D}^{*}$-related idempotents are $\rho$-related. Let $k=1$ which implies that $e_{m d+i} \rho e_{(m+1) d+i}$. Using Lemma 3.2 and the fact that $e_{m} \rho e_{n}$ implies $e_{m} \rho e_{k}$ for every $n \leq k \leq m$ together with the transitive property of the congruence, we see that the idempotents are in one $\rho$-class which is contrary to our assumption. Thus, no two distinct $\mathcal{D}^{*}$-related idempotents are $\rho$-related. Therefore $\rho$ is a *-locally idempotent-separating congruence. This completes the proof.

A typical *-idempotent-separating congruence of a *-simple type A I-semigroup is characterized in the theorem.

Theorem 3.4. Let $S=G B R^{*}(T, \theta)$ where $T=\cup_{i=0}^{d-1} M_{i}$. The relation $\rho$ on $S=G B R^{*}(T, \theta)$ defined by the rule:

$$
\left(x_{i}, m, n\right) \rho\left(y_{j}, p, q\right) \text { if and only if } m=p, n=q, i=j \text { and }\left(x_{i}, y_{j}\right) \in \operatorname{ker} \theta
$$

is a *-locally idempotent separating congruence

Proof. It can be easily shown that $\rho$ is reflexive and symmetric. To show transitivity, we let $\left(x_{i}, m, n\right) \rho\left(y_{j}, p, q\right)$, $\left(y_{j}, p, q\right) \rho\left(z_{k}, u, v\right)$ for all $\left(x_{i}, m, n\right),\left(y_{j}, p, q\right),\left(z_{k}, u, v\right) \epsilon S$. Then $m=p, n=q, i=j,\left(x_{i}, y_{j}\right) \epsilon \operatorname{ker} \theta$ and $p=u, q=$ $v, j=k,\left(y_{j}, z_{k}\right) \epsilon \operatorname{ker} \theta$.

Consequently, $m=u, n=v, i=k$. Hence $\left(x_{i}, z_{k}\right) \epsilon \operatorname{ker} \theta$, which means that $\rho$ is transitive.

Next is to show that $\rho$ is a congruence. Now let $a=\left(x_{i}, m, n\right), b=\left(y_{j}, p, q\right)$. That $\rho$ is a congruence entails showing that

$$
\begin{array}{ll}
a \rho b \text { implies } a g \rho b g & \text { (for right congruence) } \\
a \rho b \text { implies } g a \rho g b & \text { (for left congruence) }
\end{array}
$$

$\forall g=\left(z_{k}, w, l\right) \epsilon S=G B R^{*}(T, \theta)$.

Consequently,

$$
\begin{aligned}
a g & =\left(x_{i}, m, n\right)\left(z_{k}, w, l\right) \\
& = \begin{cases}\left(x_{i} \cdot f_{n-w, w}^{-1} \cdot z_{k} \theta^{n-w} \cdot f_{n-w, l}, m, n+l-w\right) & \text { if } n \geq w \\
\left(f_{w-n, m}^{-1} \cdot x_{i} \theta^{w-n} \cdot f_{w-n, n} \cdot z_{k}, m+w-n, l\right) & \text { if } n \leq w\end{cases} \\
b g & =\left(y_{j}, p, q\right)\left(z_{k}, w, l\right) \\
& = \begin{cases}\left(y_{j} \cdot f_{q-w, w}^{-1} \cdot z_{k} \theta^{q-w} \cdot f_{q-w, l}, p, q+l-w\right) & \text { if } q \geq w \\
\left(f_{w-q, p}^{-1} \cdot y_{j} \theta^{w-n} \cdot f_{w-q, q} \cdot z_{k}, p+w-q, l\right) & \text { if } q \leq w\end{cases}
\end{aligned}
$$

So, if $\left(x_{i}, m, n\right) \rho\left(y_{j}, p, q\right)$, then

$$
\left(x_{i}, m, n\right)\left(z_{k}, w, l\right) \rho\left(y_{j}, p, q\right)\left(z_{k}, w, l\right)=
$$




$$
\begin{gathered}
\begin{cases}\left(x_{i} \cdot f_{n-w, w}^{-1} \cdot z_{k} \theta^{n-w} \cdot f_{n-w, l}, m, n+l-w\right) & \text { if } n \geq w \\
\left(f_{w-n, m}^{-1} \cdot x_{i} \theta^{w-n} \cdot f_{w-n, n} \cdot z_{k}, m+w-n, l\right) & \text { if } n \leq w\end{cases} \\
\rho \begin{cases}\left(y_{j} \cdot f_{q-w, w}^{-1} \cdot z_{k} \theta^{q-w} \cdot f_{q-w, l}, p, q+l-w\right) & \text { if } q \geq w \\
\left(f_{w-q, p}^{-1} \cdot y_{j} \theta^{w-q} \cdot f_{w-q, q} \cdot z_{k}, p+w-q, l\right) & \text { if } q \leq w\end{cases}
\end{gathered}
$$

But $\left(x_{i}, m, n\right) \rho\left(y_{j}, p, q\right)$ if and only if $m=p, n=q, i=j$ and $\left(x_{i}, y_{j}\right) \epsilon \operatorname{ker} \theta$.

Thus,

$$
\begin{gathered}
\begin{cases}\left(x_{i} \cdot f_{n-w, w}^{-1} \cdot z_{k} \theta^{n-w} \cdot f_{n-w, l}, m, n+l-w\right) & \text { if } n \geq w \\
\left(f_{w-n, m}^{-1} \cdot x_{i} \theta^{w-n} \cdot f_{w-n, n} \cdot z_{k}, m+w-n, l\right) & \text { if } n \leq w\end{cases} \\
\rho \begin{cases}\left(y_{j} \cdot f_{n-w, w}^{-1} \cdot z_{k} \theta^{n-w} \cdot f_{n-w, l}, m, n+l-w\right) & \text { if } n \geq w \\
\left(f_{w-n, m}^{-1} \cdot y_{j} \theta^{w-n} \cdot f_{w-n, n} \cdot z_{k}, m+w-n, l\right) & \text { if } n \leq w\end{cases}
\end{gathered}
$$

Hence $\rho$ is a right congruence.

That $\rho$ is a left congruence follows similarly. Thus $\rho$ is a congruence.

Furthermore, $\left(e_{i}, m, m\right) \rho\left(e_{i}, n, n\right)$ implies $m=n$ which implies $\left(e_{i}, m, m\right)=\left(e_{i}, n, n\right)$. Thus any two distinct idempotent elements which are $\mathcal{D}^{*}$-related cannot lie in the same $\rho$-class. Hence the proof.

We will now construct the strictly *-locally idempotent-separating congruences on *-simple type A Isemigroups.

3.5. Notation. Let $k_{0}, k_{1}, k_{2}, k_{3}, \ldots, k_{t}$ be a sequence of non-empty integers, satisfying $0 \leq k_{0}<k_{1} \ldots<k_{t}<$ $d-1, k_{0}=-1, k_{t+1}=d-1$.

Define a relation $\rho=\rho\left(k_{0}, k_{1}, \ldots, k_{t}\right)$ on $S=G B R^{*}(T, \theta)$ by

$\left(x_{i}, m, n\right) \rho\left(y_{j}, p, q\right)$ implies $\left\{\begin{array}{l}m=p, n=q \text { for } k_{v-1}<i, j \leq k_{v}, 0 \leq v \leq t+1 \\ \text { or } m=p+1, n=q+1 \text { for } i \leq k_{0} \text { and } j>k_{t} \\ \text { or } m+1=p, n+1=q \text { for } j \leq k_{0} \text { and } i>k_{t}\end{array}\right.$

Lemma 3.6. With the notation introduced, $\rho=\rho\left(k_{0}, k_{1}, \ldots, k_{t}\right)$ is a strictly *-locally idempotent-separating congruence on $S=G B R^{*}(T, \theta)$.

Proof. Suppose $\rho$ is a strictly *-locally idempotent-separating congruence on $S=G B R^{*}(T, \theta)$. Then we have that

$$
\left(x_{i}, m, n\right) \rho\left(y_{j}, p, q\right) \text { implies }\left(y_{j}, n, m\right) \rho\left(x_{i}, q, p\right)
$$

since it is evident that the relation $\rho$ defined above is a congruence on a type $A$ semigroup (where $\left(y_{j}, n, m\right)$ is inverse of $\left(x_{i}, m, n\right)$ and $\left(x_{i}, q, p\right)$ is the inverse of $\left(y_{j}, p, q\right)$ ).

Now we have that $\left(x_{i}, m, n\right)^{\dagger} \rho\left(y_{j}, p, q\right)^{\dagger}$ and $\left(x_{i}, m, n\right)^{*} \rho\left(y_{j}, p, q\right)^{*}$ implies $\left(e_{i}, m, m\right) \rho\left(e_{i}, p, p\right)$ and $\left(e_{i}, n, n\right) \rho\left(e_{i}, q, q\right)$.

That is, we have that $e_{m d+i} \rho e_{p d+j}$ and $e_{n d+i} \rho e_{q d+j}$.

Suppose $m d+i \geq(p+1) d+j$ then $e_{p d+j} \rho e_{(p+1) d+j}$ then $i<j, m \leq p+1 . \quad i>j, m \leq p$. 
Similarly, we have $j<i, p \leq m+1 . \quad j>i, p \leq m$.

Consequently, we have $i<j, m \leq p+1 \leq m+1$. That is $m=p$ or $p+1$.

$i>j, m \leq p \leq m+1$. That is $p=m$ or $m+1$.

Interchanging the roles of $m$ and $n, \mathrm{p}$ and $q$ we have that

$i<j, n=q$ or $q+1 . \quad i>j, q=n$ or $n+1$.

Now using Lemma 3.2 and considering some cases, we have the desired result.

We now consider cancellative monoid congruences. These can be characterized as follows:

Theorem 3.7. Let $S=G B R^{*}(T, \theta)$ be a *-simple type A /-semigroup. Define a relation $\sigma$ on $S$ by

$$
\left(x_{i}, m, n\right) \sigma\left(y_{j}, p, q\right)
$$

if and only if $m-n=p-q$ and $x_{i}=y_{j}$. Then

i) $\sigma$ is the minimum congruence on $S$.

ii) $S / \sigma$ is a cancellative monoid.

Proof. i) That $\sigma$ is reflexive and symmetric can be easily checked. To show transitivity, let $\left(x_{i}, m, n\right) \sigma\left(y_{j}, p, q\right)$ and $\left(y_{j}, p, q\right) \sigma\left(z_{k}, r, c\right)$ for $\left(x_{i}, m, n\right),\left(y_{j}, p, q\right),\left(z_{k}, r, c\right) \in S$. Then we have $m-n=p-q, x_{i}=y_{j}$ and $p-q=$ $r-c, y_{j}=z_{k}$. This implies $m-n=r-c$ and $x_{i}=z_{k}$. Thus $\sigma$ is transitive.

Now let $a=\left(x_{i}, m, n\right), b=\left(y_{j}, p, q\right)$. That $\sigma$ is a congruence entails showing that

$$
\begin{gathered}
a \sigma b \Rightarrow a u \sigma b u \quad \text { (for right congruence) } \\
a \sigma b \Rightarrow u a \sigma u b \quad \text { (for left congruence) }
\end{gathered}
$$

$\forall u=\left(z_{k}, r, c\right) \in S$. So, we have that

$$
\begin{gathered}
a u=\left(x_{i}, m, n\right)\left(z_{k}, r, c\right)= \begin{cases}\left(x_{i} \cdot f_{n-r, r}^{-1} \cdot z_{k} \theta^{n-r} \cdot f_{n-r, c}, m, n+c-r\right) & \text { if } n \geq r \\
\left(f_{r-n, n}^{-1} \cdot x_{i} \theta^{r-n} \cdot f_{r-n, n} \cdot z_{k}, m+r-n, c\right) & \text { if } n \leq r\end{cases} \\
b u=\left(y_{j}, p, q\right)\left(z_{k}, r, c\right)= \begin{cases}\left(y_{j} \cdot f_{q-r, r}^{-1} \cdot z_{k} \theta^{q-r} \cdot f_{q-r, c}, p, q+c-r\right) & \text { if } q \geq r \\
\left(f_{r-q, q}^{-1} \cdot y_{j} \theta^{r-q} \cdot f_{r-q, q} \cdot z_{k}, p+r-q, c\right) & \text { if } q \leq r\end{cases}
\end{gathered}
$$

Suppose $\left(x_{i}, m, n\right) \sigma\left(y_{j}, p, q\right)$, we have

$$
\begin{gathered}
m-(n+c-r)=(m-n)+(r-c) \text { and } p-(q+c-r)=(p-q)+(r-c) \\
m+r-n-c=(m-n)+(r-c) \text { and } p+r-q-c=(p-q)+(r-c) .
\end{gathered}
$$

Since $m-n=p-q$, we have that $(m-n)+(r-c)=(p-q)+(r-c)$.

Consequently, $\sigma$ is a right congruence. That $\sigma$ is a left congruence follows similarly. Thus $\sigma$ is a congruence. 
Suppose $\rho$ is any other congruence. Then we have $(1, m, m) \rho(1,0,0)$ for all $m \in I$. If $\left(x_{i}, m, n\right) \sigma\left(y_{j}, p, q\right)$, then $\left(x_{i}, m, n\right)(1, p, p)=\left(y_{j}, p, q\right)(1, p, p)$ for some $p \in I$

Since $(1, m, m) \rho(1,0,0)$, then $\left(x_{i}, m, n\right)(1, p, p) \rho\left(x_{i}, m, n\right)$.

Similarly, $\left(y_{j}, p, q\right)(1, p, p) \rho\left(y_{j}, p, q\right)$ so that $\left(x_{i}, m, n\right) \rho\left(y_{j}, p, q\right)$. Hence $\sigma \subseteq \rho$.

ii) Obviously the class of $\sigma$ containing the idempotents is the identity element for $S / \sigma$. So we have $(1, m, n) \sigma\left(y_{j}, p, q\right) \sigma=\left(y_{j}, p, q\right) \sigma$. Thus $S / \sigma$ is a monoid.

To show that $S / \sigma$ is cancellative, let $a=\left(x_{i}, m, n\right), b=\left(y_{j}, p, q\right)$. That $S / \sigma$ is cancellative entails showing that

$$
\begin{array}{ll}
a \sigma u \sigma=b \sigma u \sigma \Rightarrow a \sigma=b \sigma & \text { (for right cancellative) } \\
u \sigma a \sigma=u \sigma b \sigma \Rightarrow a \sigma=b \sigma & \text { (for left cancellative) }
\end{array}
$$

$\forall u=\left(z_{k}, r, c\right) \in S$. So, we have that

$$
\begin{aligned}
a \sigma u \sigma & =\left(x_{i}, m, n\right) \sigma\left(z_{k}, r, c\right) \sigma=\left(y_{j}, p, q\right) \sigma\left(z_{k}, r, c\right) \sigma \\
& =b \sigma u \sigma .
\end{aligned}
$$

The rest of the proof follows from a routine calculation.

For the remainder of this section the group of integers under addition will be denoted by $\mathbb{Z}$.

We now describe the nature of $S / \sigma$ in the case where $\theta$ is the identity mapping.

Theorem 3.8. Let $S=G B R^{*}(T, \theta)$ be a *-simple type A I-semigroup in which $\theta$ is the identity mapping. Define a multiplication on the set $T \times \mathbb{Z}$ by the rule that

$$
\left(x_{i}, m d+i\right)\left(y_{j}, n d+i\right)=\left(x_{i} y_{j},(m d+i)+(n d+i)\right)
$$

for $x_{i}, y_{j} \in T, m, n \in \mathbb{Z}$. Then $S / \sigma \cong T \times \mathbb{Z}$.

Proof. Define a map $\varphi: S \rightarrow T \times \mathbb{Z}$ by the rule that $\left(x_{i}, m, n\right) \varphi=\left(x_{i} y_{j},(m d+i)-(n d+i)\right)$.

Evidently, $\varphi$ is well defined. It is known that $T \times \mathbb{Z}$ is a cancellative monoid with identity $(1,0)$.

Now let $\left(x_{i}, m, n\right)$ and $\left(y_{j}, p, q\right)$ be any two elements of $S$. Then

$$
\begin{aligned}
& \left(\left(x_{i}, m, n\right)\left(y_{j}, p, q\right)\right) \varphi=\left\{\begin{array}{ll}
\left(x_{i} \cdot f_{n-p, p}^{-1} \cdot y_{j} \theta^{n-p} \cdot f_{n-p, q}, m, n+q-p\right) & \text { if } n \geq p \\
\left(f_{p-n, n}^{-1} \cdot x_{i} \theta^{p-n} \cdot f_{p-n, n} \cdot y_{j}, m+p-n, q\right) & \text { if } n \leq p
\end{array} \times \varphi\right. \\
& = \begin{cases}\left(x_{i} \cdot f_{n-p, p}^{-1} \cdot y_{j} \theta^{n-p} \cdot f_{n-p, q}, m, n+q-p\right) \varphi & \text { if } n \geq p \\
\left(f_{p-n, n}^{-1} \cdot x_{i} \theta^{p-n} \cdot f_{p-n, n} \cdot y_{j}, m+p-n, q\right) \varphi & \text { if } n \leq p\end{cases} \\
& = \begin{cases}\left(x_{i} y_{j}, m d+i-(n+q-p) d+i\right) & \text { if } n \geq p \\
\left(x_{i} y_{j},(m+p-n) d+j-q d+j\right) & \text { if } n \leq p\end{cases}
\end{aligned}
$$




$$
\begin{aligned}
& =\left\{\begin{array}{ll}
\left(x_{i} y_{j},(m-n) d+i+(p-q) d+i\right) \\
\left(x_{i} y_{j},(m-n) d+j+(p-q) d+j\right)
\end{array} \text { if } n \geq p\right. \\
& =\left(x_{i} y_{j},(m-n) d+i+(p-q) d+j\right) \\
& =\left(x_{i},(m-n) d+i\right)\left(y_{j},(p-q) d+j\right) \\
& =\left(x_{i},(m d+i)-(n d+i)\right)\left(y_{j},(p d+j)-(q d+j)\right) \\
& =\left(x_{i}, m, n\right) \varphi\left(y_{j}, p, q\right) \varphi .
\end{aligned}
$$

Thus $\varphi$ is a homomorphism.

Furthermore,

$$
\left(x_{i}, m, n\right) \varphi=\left(y_{j}, p, q\right) \varphi
$$

if and only if $\left(x_{i},(m d+i)-(n d+i)\right)=\left(y_{j},(p d+j)-(q d+j)\right)$

if and only if $(m d+i)-(n d+i)=(p d+j)-(q d+j)$ and $x_{i}=y_{j}$

if and only if $\left(x_{i}, m, n\right) \sigma=\left(y_{j}, p, q\right) \sigma$.

That is $\varphi \circ \varphi^{-1}=\sigma$.

\section{References}

1. $\quad$ Asibong-Ibe, U.I. *-Simple type $A \omega$-semigroups, Semigroup Forum 47 (1993), 135-149.

2. Fountain, J.B. A class of right PP monoids, Quart. J. Math. Oxford 2, 28 (1974), 28-44.

3. Fountain, J.B. Adequate semigroups. Proc. Edinburgh Math. Soc 22 (1979), 113-125.

4. Fountain, J.B. Abundant semigroups, Proc. London. Math. Soc., (3) 44 (1982), 103-129.

5. Howie, J.M. Fundamentals of Semigroup Theory, Oxford University Press, Inc. USA, 1995.

6. Makanjuola, S. O. Congruences on type A $\omega$-semigroups. D.Phil Thesis, Ahmadu Bello Univer.,1988.

7. Ndubuisi, R.U and Asibong-Ibe, U.I. Congruences on *-bisimple type A I-semigroup. Journal of Semigroup Theory and Applications, Vol. 2018, Article ID 4 (2018), 1-14.

8. Ndubuisi, R.U, Asibong-Ibe, U.I and UdoAkpan, I.U. A class of *-simple type A I-semigroups. Int'1 J. Mathematics and its applications, 6(1-E) (2018), 1227-1234.

9. Shang, Y. and Wang, L. *-Bisimple type $A$ I-semigroups, Southeast Asian Bull. Math. 36 (2012), 535545 .

10. Warne, R.J. Some properties of simple I-regular semigroup. Compositio Math. Vol 22 (1970), 181195. 